\title{
Experimental analysis of the feasibility of low-cost piezoelectric diaphragms in impedance-based SHM applications
}

\author{
Everaldo Silva de Freitas ${ }^{\mathrm{a}, \mathrm{b}}$, Fabricio Guimarães Baptista ${ }^{\mathrm{a}, *}$ \\ a Faculdade de Engenharia, UNESP-Univ Estadual Paulista, Campus de Bauru, Departamento de Engenharia Elétrica, 17033-360 Bauru, SP, Brazil \\ b Centro Universitário de Lins, Unilins, Departamento de Engenharia Elétrica e Automação, 16401-371 Lins, SP, Brazil
}

\section{A R T I C L E I N F O}

\section{Article history:}

Received 14 August 2015

Received in revised form

17 November 2015

Accepted 27 November 2015

Available online 24 December 2015

\section{Keywords:}

Piezoelectric diaphragms

PZT

SHM

Impedance

Damage detection

Feasibility

\begin{abstract}
A B S T R A C T
Structural health monitoring (SHM) systems with damage detection based on the electromechanical impedance (EMI) method have received attention in recent decades because of the simplicity of the methodology, which is based on the use of small and lightweight piezoelectric transducers that operate simultaneously as sensors and actuators. Typically, the piezoelectric transducers used in the EMI method have been thin PZT (lead zirconate titanate) ceramics, which can be cut into different sizes and shapes. In this study, an experimental analysis of the feasibility of low-cost piezoelectric diaphragms, commonly known as buzzers, for damage detection based on the EMI method is presented. Diaphragms of different sizes were evaluated in aluminum beams, and the experimental results were compared with those obtained from a conventional PZT ceramic. Four types of experiments were performed: sensitivity estimation using the pencil lead break (PLB) method, analysis of the ability to detect structural damage using conventional impedance signatures and damage indices, analysis of temperature effects, and determination of the long-term reproducibility of the results. The results indicate that a conventional PZT ceramic and a diaphragm of similar size have similar reproducibilities and sensitivities to damage and temperature effects.
\end{abstract}

(C) 2015 Elsevier B.V. All rights reserved.

\section{Introduction}

Piezoelectric diaphragms, which are commonly known as buzzers, are simple acoustic components that are widely used in a variety of electronic devices to produce a sound, such as an alarm, beep, or ringing. In this study, we performed an experimental analysis of the feasibility of these acoustic components for detecting structural damage in structural health monitoring (SHM) applications based on the electromechanical impedance (EMI) principle.

SHM systems [1,2] are able to detect incipient structural damage, thereby increasing safety and reducing maintenance costs. Examples of the various structures that can be monitored include bridges, aeronautical structures, pipelines, dams and oil platforms. The detection of damage should be performed using a minimally invasive method, known as nondestructive evaluation (NDE) or nondestructive testing (NDT) [3]. There are many methods for detecting damage and other changes in structure, such as acoustic emission (AE) [4], eddy current [5], Lamb waves [6], methods

\footnotetext{
* Corresponding author.

E-mail addresses: fabriciogb@feb.unesp.br, fabriciogb@ieee.org (F. Guimarães Baptista).
}

based on fiber-optic sensors [7], and comparative vacuum [8]. In this study, we focused on damage detection based on the EMI method, which uses small, lightweight piezoelectric transducers that are adhered to the monitored structure.

The EMI method [9-12] is based on the piezoelectric effect. When a piezoelectric transducer is fastened to a structure, an interaction occurs between the mechanical properties of the structure and the electrical impedance of the transducer. Therefore, it is possible to detect changes in the mechanical properties of the structure caused by damage simply by measuring the electrical impedance of the transducer.

The transducers used in EMI and other NDT methods have commonly been PZT (lead zirconate titanate) ceramics [6,13,14], also known as piezoelectric wafer active sensors (PWAS) [15], which are typically fine ceramics coated on both sides with thin metal films that act as electrodes. Our objective in this study is to experimentally assess the feasibility of piezoelectric diaphragms compared with conventional PZT ceramics. The piezoelectric diaphragms (or buzzers) have a simple circular construction consisting of a brass plate onto which a piezoelectric ceramic disc is fixed. These acoustic components are readily obtained and inexpensive, which make them attractive for other applications besides producing sound. 
Several scientific studies have used piezoelectric diaphragms as sensors or actuators in various applications. For example, they have been successfully used as the frictional element and mechanical vibrator in a piezoelectric clutch mechanism [16], as active elements in acoustic positioning (track-pad) applications [17], as sensing elements in a pressure sensor design [18], as ultrasonic actuators [19], and as generators of electricity in energy-harvesting applications [20]. Diaphragm buzzers have also been used for damage detection in SHM applications. In [21], the authors used eight piezoelectric diaphragms for damage detection in an aeronautical panel based on the EMI method. A single buzzer has been used in [22] to detect damage in aluminum beams using the EMI method. In addition, the authors conducted a simple comparison between the results obtained using the buzzer and a conventional PZT ceramic. Sets of two piezoelectric diaphragms were used in [23] to identify structural damage in active plates with indices based on Gaussian confidence ellipses of the electromechanical admittance. Damage detection based on other methods using low-cost diaphragms has also been reported [24,25], indicating the feasibility of these simple acoustic components.

Although these studies reported the feasibility of piezoelectric diaphragms for structural damage detection, a more detailed analysis and comparison with conventional transducers is still required, especially for the EMI method. Therefore, in this study, we performed a detailed experimental analysis of the feasibility of piezoelectric diaphragms for detecting damage based on the EMI method. Tests were performed on aluminum beams, and piezoelectric diaphragms of different sizes were analyzed to determine their sensitivities to structural damage and temperature effects as well as the reproducibility of the results. Furthermore, the results obtained using the diaphragms were compared with those obtained using a conventional PZT ceramic.

Our paper is organized as follows: The background of the EMI method is presented in Section 2. Section 3 describes the piezoelectric diaphragms and the conventional PZT ceramic device used in this study. The experimental setup is shown in Section 4. Section 5 presents the experimental results. The article ends with the conclusions in Section 6 and the references.

\section{Electromechanical impedance (EMI) method}

The detection of structural damage based on the EMI method is unique because it has a very simple methodology. Fig. 1 shows the basic configuration of the method in which a PZT patch is attached to the structure to be monitored.

A measuring system excites the patch and measures its electrical impedance $\left(Z_{E}(\omega)\right)$ over a suitable frequency range. Therefore, in the EMI method, the piezoelectric transducer operates simultaneously as a sensor (direct piezoelectric effect) and actuator (reverse piezoelectric effect). The direct and reverse linear piezoelectric effects are given by Eqs. (1) and (2), respectively, as follows [26]:

$$
\begin{aligned}
& D_{i}=d_{i k l} T_{k l}+\epsilon_{i k}^{T} E_{k} \\
& S_{i j}=s_{i j k l}^{E} T_{k l}+d_{k i j} E_{k}
\end{aligned}
$$

where $E_{k}$ and $D_{i}$ are the components of the electric field intensity and electric displacement, respectively; $d_{i k l}$ and $d_{k i j}$ are the piezoelectric constants; $s_{i j k l}^{E}$ are the elastic compliance constants at constant electric field; $\epsilon_{i k}^{T}$ are the dielectric constants at constant

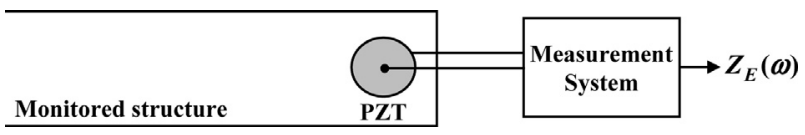

Fig. 1. Basic configuration of the EMI method. stress; $T_{k l}$ and $S_{i j}$ are the stress and strain components, respectively; and $i, j, k$, and $l$ have values of 1,2 , and 3 representing the axes of the natural coordinate system of the piezoelectric material.

Based on the piezoelectric constitutive equations shown in Eqs. (1) and (2), it is possible to relate the electrical impedance of a PZT patch, as shown in Fig. 1, to the mechanical impedance of the monitored structure. Many electromechanical models [27] have been proposed to relate the electrical and mechanical quantities. A pioneering study was conducted by Liang et al. [28]. in which the electrical impedance of the PZT patch considering a onedimensional assumption is given by

$Z_{E}(\omega)=\frac{1}{j \omega a}\left(\epsilon_{33}^{T}-\frac{Z_{S}(\omega)}{Z_{E}(\omega)+Z_{P}(\omega)} \frac{d_{31}^{2}}{s_{11}^{E}}\right)^{-1}$

where $Z_{E}(\omega)$ is the electrical impedance of the PZT patch at angular frequency $\omega$, which should vary within an appropriate range; $Z_{P}(\omega)$ and $Z_{S}(\omega)$ are the mechanical impedances of the PZT patch and the monitored structure, respectively; a is a geometric constant that depends on the shape and size of the patch; $j$ is the unit imaginary number; and $\epsilon_{33}^{T}, \mathrm{~d}_{31}$ and $s_{11}^{E}$ are the same as defined before considering a one-dimensional assumption.

According to Eq. (3), there is a relationship between the electrical impedance $Z_{E}(\omega)$ of the PZT patch and the mechanical impedance $Z_{S}(\omega)$ of the monitored structure. Variations in the mechanical impedance caused by damage or other alterations in the structure, such as cracks, corrosion, and loosening of bolted connections, cause corresponding variations in the electrical impedance of the PZT patch. Therefore, the structure can be monitored and damage can be detected by analyzing the electrical impedance of the patch, which is easily measured.

The electrical impedance obtained from the PZT patch is complex, with a real part (resistance) and an imaginary part (reactance). Typically, the real part is used for structural monitoring because it is more sensitive to damage or changes in the structure's integrity [29]. The damage detection is performed using a damage index, which compares two electrical impedance signatures over an appropriate frequency range. One of these signatures is obtained when the structure is healthy and used as a reference, known as the baseline. One of the damage indices most widely used in the EMI method is the root-mean-square deviation (RMSD), which is given by [1]

$\operatorname{RMSD}=\sum_{\omega=\omega_{I}}^{\omega_{F}} \sqrt{\frac{\left[\operatorname{Re}\left(Z_{2}(\omega)\right)-\operatorname{Re}\left(Z_{1}(\omega)\right)\right]^{2}}{\operatorname{Re}\left(Z_{1}(\omega)\right)^{2}}}$

where $\operatorname{Re}\left(Z_{1}(\omega)\right)$ is the real part of the baseline signature, $\operatorname{Re}\left(Z_{2}(\omega)\right)$ is the real part of the impedance signature after possible damage, and RMSD is the index calculated in the frequency range with initial frequency $\omega_{I}$ and final frequency $\omega_{F}$.

In the past, impedance measurements were typically performed with expensive commercial impedance analyzers, which have many features not required for the EMI method. Many researchers have proposed alternative measurement systems [12,30,31] to simplify the measurement of impedance and lower its cost.

The piezoelectric diaphragms and the conventional PZT ceramic used in this study are presented in the next section.

\section{Piezoelectric diaphragms}

PZT ceramics have traditionally been the most common transducers used in the EMI method. These ceramics are thin, typically ranging from 0.1 to $2 \mathrm{~mm}$ in thickness. These devices are usually coated on both sides with metal films functioning as electrodes, and they are produced in different sizes and shapes. Our proposal was to experimentally evaluate the feasibility of the use of piezo- 
Table 1

Dimensions and properties of the piezoelectric diaphragms.

\begin{tabular}{|c|c|c|c|c|c|c|c|}
\hline \multirow[t]{2}{*}{ Model } & \multicolumn{2}{|l|}{ Brass plate } & \multicolumn{5}{|l|}{ Active element } \\
\hline & Diameter(mm) & Thickness(mm) & Diameter(mm) & Thickness(mm) & $d_{31}\left(10^{-12} \mathrm{~m} / \mathrm{V}\right)$ & $s_{11}^{E}\left(10^{-12} \mathrm{~m}^{2} / \mathrm{N}\right)$ & $\epsilon_{33}^{T} / \epsilon_{0}$ \\
\hline 7BB-12-9 & 12.0 & 0.10 & 9.0 & 0.12 & -207 & 15.8 & 2100 \\
\hline 7BB-20-6 & 20.0 & 0.20 & 14.0 & 0.22 & & & \\
\hline 7BB-27-4 & 27.0 & 0.30 & 19.7 & 0.24 & & & \\
\hline 7BB-35-3 & 35.0 & 0.30 & 25.0 & 0.23 & & & \\
\hline
\end{tabular}

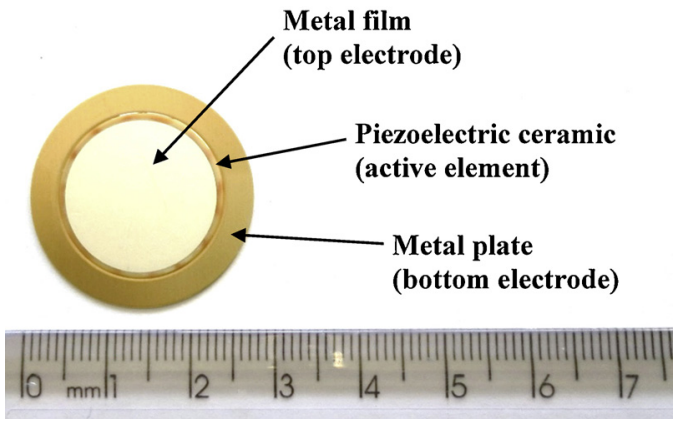

Fig. 2. Piezoelectric diaphragm.

electric diaphragms (buzzers) in the EMI method; as mentioned previously, these diaphragms have been used in various scientific studies [16-25].

Piezoelectric diaphragms have a very simple construction. They consist of a circular piezoelectric ceramic (the active element) mounted on a circular metal plate (the diaphragm). The ceramic is coated with a thin, metallic film (usually silver) that serves as an electrode. Typically, the piezoelectric material is barium titanate or PZT (not pure but doped), and the metal diaphragm is available in brass (most commonly), nickel alloy, or stainless steel. Fig. 2 shows a typical diaphragm and descriptions of its parts.

Four diaphragms of different sizes from Murata Electronics North America, Inc. (Smyrna, GA, USA) [32] were evaluated in this study; the external diameters (brass plate diameter) ranged from 12 to $35 \mathrm{~mm}$, and the active element was a type P-7 PZT ceramic. A conventional PZT-5A ceramic from Piezo Systems (Woburn, MA, USA) [33] was used as a reference for comparison of the results. The dimensions and properties of the diaphragms and conventional ceramic are presented in Tables 1 and 2, respectively, where $\epsilon_{33}^{T} / \epsilon_{0}$ is the relative dielectric constant and $\varepsilon_{0}$ is the vacuum permittivity.

Fig. 3 shows (a) a comparison between the 7BB-20-6 diaphragm $(20 \mathrm{~mm})$ and the conventional ceramic and (b) the different diaphragms evaluated.

As apparent from Fig. 3(a) and Tables 1 and 2, the 7BB-20-6 diaphragm has an active element with dimensions and properties similar to those of the conventional ceramic. Therefore, this configuration allows for a direct comparison between the two transducers and an evaluation of the influence of the brass plate of the piezoelectric diaphragms on the results. In addition to the direct comparison between the 7BB-20-6 diaphragm and the conventional ceramic, diaphragms of different sizes were also compared. The experimen-

Table 2

Dimensions and properties of the conventional PZT ceramic.

\begin{tabular}{ll}
\hline Model & T107-A4E-273 \\
\hline Diameter $(\mathrm{mm})$ & 12.7 \\
Thickness $(\mathrm{mm})$ & 0.191 \\
$d_{31}\left(10^{-12} \mathrm{~m} / \mathrm{V}\right)$ & -190 \\
$s_{11}^{E}\left(10^{-12} \mathrm{~m}^{2} / \mathrm{N}\right)$ & 15.2 \\
$\epsilon_{33}^{T} / \epsilon_{0}$ & 1800 \\
\hline
\end{tabular}

tal procedure used to evaluate these transducers is presented in the next section.

\section{Experimental setup}

To assess the feasibility of the diaphragms and compare the results with those obtained using the conventional ceramic, tests were performed on $500 \times 38.10 \times 3.18 \mathrm{~mm}$ aluminum beams. The transducers were bonded to the beams $30 \mathrm{~mm}$ from the beam ends using cyanoacrylate glue. The transducers installed on the aluminum beams are shown in Fig. 4.

Four types of tests were performed: assessment of the sensitivity to damage using the pencil lead break (PLB) method, assessment of structural damage detection via the EMI method based on the electrical impedance signatures and the RMSD index, analysis of the temperature effects, and long-term reproducibility.

The sensitivity of the transducers to structural damage was assessed using the PLB method [34,35], which is a formal methodology adopted as a standard [36] for the generation of an acoustic emission source. The test consists of breaking a pencil lead against a structure or support on which the sensor is installed. The breaking of the lead releases an impulsive stress and an elastic wave with a wide frequency spectrum, which can be used to assess the piezoelectric transducers. We used a mechanical pencil and a lead with a diameter of $0.5 \mathrm{~mm}$ and a length of $2 \mathrm{~mm}$. The lead was broken at a distance of $100 \mathrm{~mm}$ from each transducer and an angle of approximately $40^{\circ}$ to the structure. The voltage signals from each transducer were acquired at room temperature using a data acquisition (DAQ) device (NI-USB-6361) with a sampling rate of $2 \mathrm{MS} / \mathrm{s}$ and a passive RC anti-aliasing filter with a cutoff frequency of approximately $750 \mathrm{kHz}$, allowing for reliable analysis for frequencies up to $500 \mathrm{kHz}$. The results were analyzed in the frequency domain using averaged power spectral density (PSD), which was calculated using 10 measurements of time-domain signals from each transducer.

In the second test, the transducers were evaluated using the conventional RMSD index. Structural damage was induced in the structures by adding a metal bolt (steel nut) of $8 \times 4 \mathrm{~mm}$ and a mass of approximately $1 \mathrm{~g}$ at a distance of $100 \mathrm{~mm}$ from each transducer. Adding a bolt causes a change in the mechanical impedance of the structure that can be related to damage. This procedure has the advantage of not causing permanent damage to the specimens.

The EMI method is highly sensitive to temperature variation $[29,37]$. In the third test, to evaluate the performance of the piezoelectric diaphragms at different temperatures and compare the results with those obtained using the conventional ceramic, the beams were heated using a heat gun, and the electromechanical impedance signatures were obtained for temperatures ranging from 20 to $70^{\circ} \mathrm{C}$ in steps of $2^{\circ} \mathrm{C}$.

Typically, the detection and quantification of damage is performed by comparing two impedance signatures using damage indices; thus, the reproducibility of the impedance signatures is important to avoid false-positive diagnoses. Therefore, the last test examined the reproducibility of the results obtained using the piezoelectric diaphragms by measuring the impedance signatures 


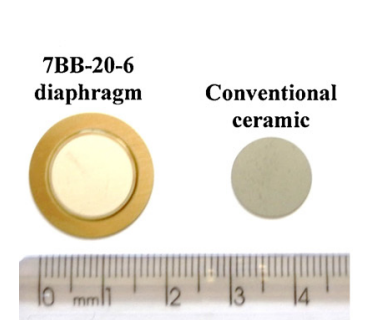

(a)

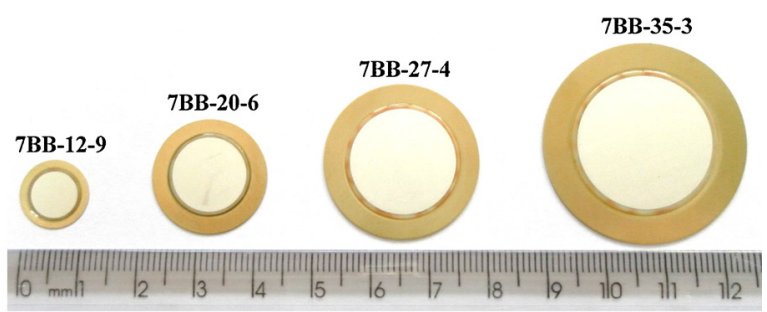

(b)

Fig. 3. (a) The 7BB-20-6 diaphragm and the conventional ceramic and (b) the diaphragms of different sizes evaluated.

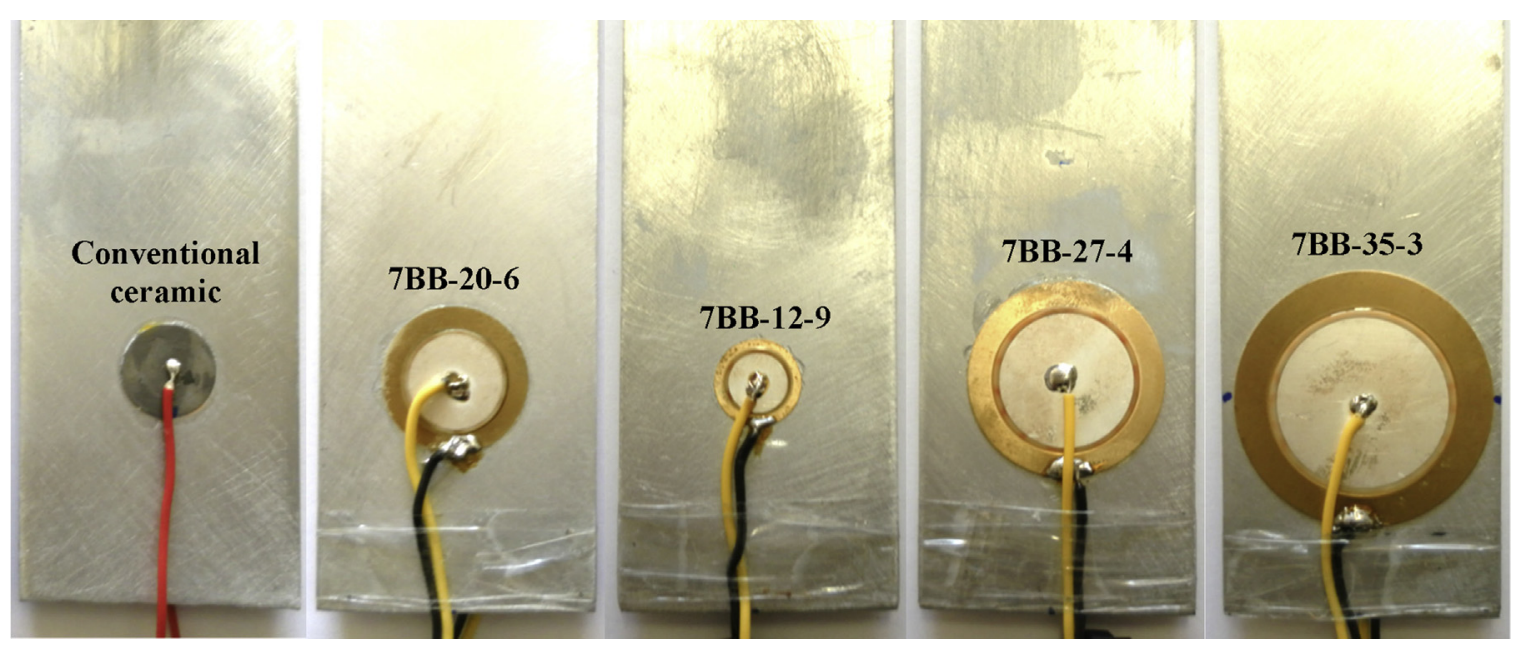

Fig. 4. Aluminum beams with the piezoelectric transducers.

every $24 \mathrm{~h}$ over one week at a temperature of $20^{\circ} \mathrm{C}$ controlled by an air conditioner.

The electrical impedance signatures were obtained using the measurement system proposed in [31] with a multifunction DAQ device (NI-USB-6361) operating at a sampling rate of $2 \mathrm{MS} / \mathrm{s}$ and a personal computer running LabVIEW (National Instruments, Austin, Texas, USA). The transducers were excited with a chirp signal at $1 \mathrm{~V}$ of amplitude, and the signatures were obtained in the frequency range of $0-500 \mathrm{kHz}$ in steps of $2 \mathrm{~Hz}$.

The results are analyzed and discussed in the next section.

\section{Results and discussion}

\subsection{Pencil lead break (PLB) test}

The signals obtained from the PLB test were analyzed in the frequency domain by computing the PSD. A comparison of the PSDs obtained from the conventional ceramic and the 7BB-20-6 diaphragm, which has a piezoelectric element of similar size, is shown in Fig. 5.

As shown in Fig. 5, the PSDs obtained from the conventional ceramic and the 7BB-20-6 diaphragm exhibit similar trends. This important result indicates that a piezoelectric diaphragm with an active element with dimensions similar to those of a conventional PZT ceramic has a similar frequency response. Comparing the two results, the conventional ceramic has a higher PSD at frequencies below approximately $15 \mathrm{kHz}$. In contrast, the diaphragm provides a higher PSD at high frequencies. However, both transducers exhibit a decreasing trend in their PSDs as the frequency increases.

The results obtained from the other diaphragms of different sizes are shown in Fig. 6.

As in the previous result, the PSDs obtained for the other diaphragm sizes show similar trends. At frequencies below approx-

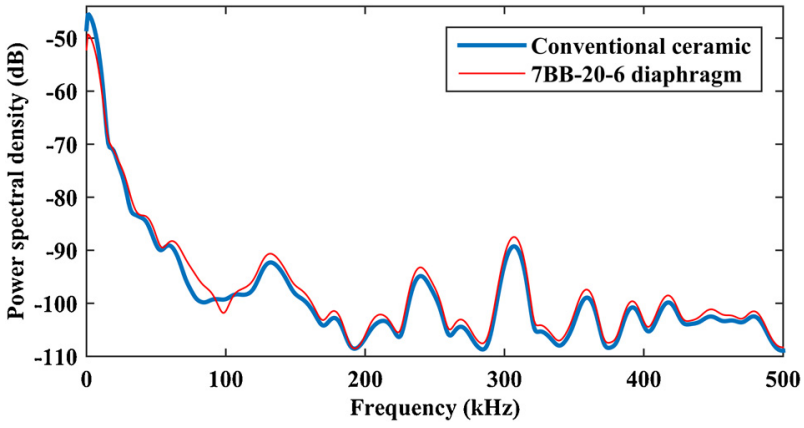

Fig. 5. PSDs obtained from the conventional ceramic and the 7BB-20-6 diaphragm used in the PLB test.

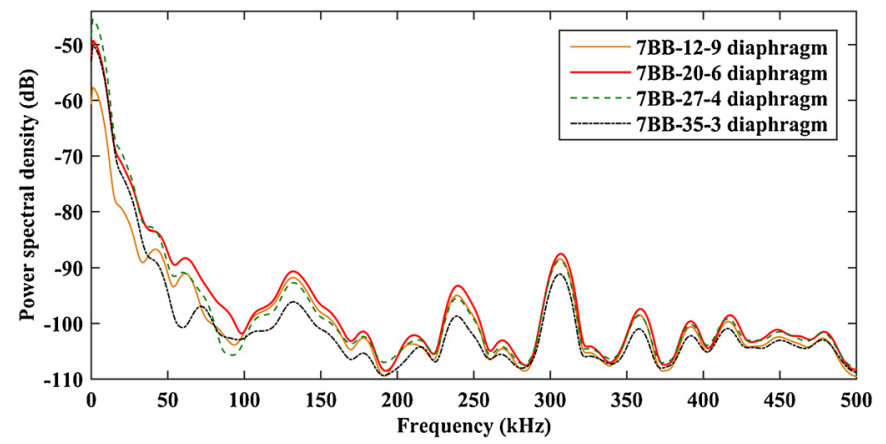

Fig. 6. PSDs obtained from the piezoelectric diaphragms of different sizes used in the PLB test.

imately $35 \mathrm{kHz}$, the smallest diaphragm (7BB-12-9) provides the lowest PSD, and the 7BB-27-4 diaphragm provides the highest PSD. 

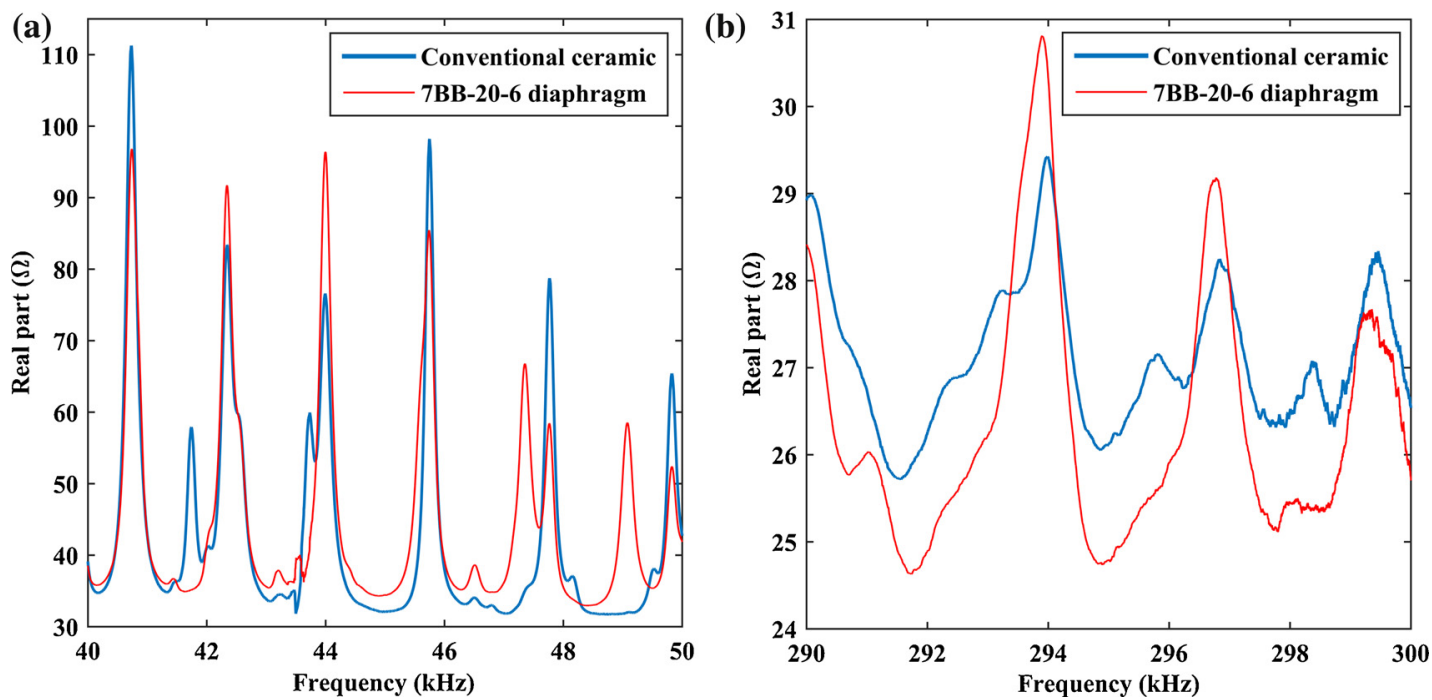

Fig. 7. Real parts of the impedance signatures of the conventional ceramic and the 7BB-20-6 diaphragm in the two sub-band frequency ranges.

However, at high frequencies, the largest diaphragm (7BB-35-3) provides the lowest PSD, and the 7BB-20-6 diaphragm provides the highest PSD.

According to the results reported in [35], the damage indices calculated using the electrical impedance signatures exhibit trends similar to those of the PSDs obtained using the PLB test. The RMSD indices obtained from the conventional ceramic and piezoelectric diaphragms for the purposes of detecting and quantifying structural damage are presented and discussed in the next section.

\subsection{Structural damage detection}

The feasibility of a transducer for detecting structural damage can be verified based on the damage indices. We used the RMSD index, which was calculated using Eq. (4), and the real part of the impedance signature was used to improve the sensitivity to damage [29].

Fig. 7 shows the real parts of the electrical impedance signatures obtained from the conventional PZT ceramic and the 7BB-20-6 piezoelectric diaphragm for the structure in a healthy condition at $30^{\circ} \mathrm{C}$. Although the impedance signatures were measured over the wide frequency range of $0-500 \mathrm{kHz}$, two sub-bands, i.e., (a) $40-50 \mathrm{kHz}$ and (b) $290-300 \mathrm{kHz}$, are shown for comparison.

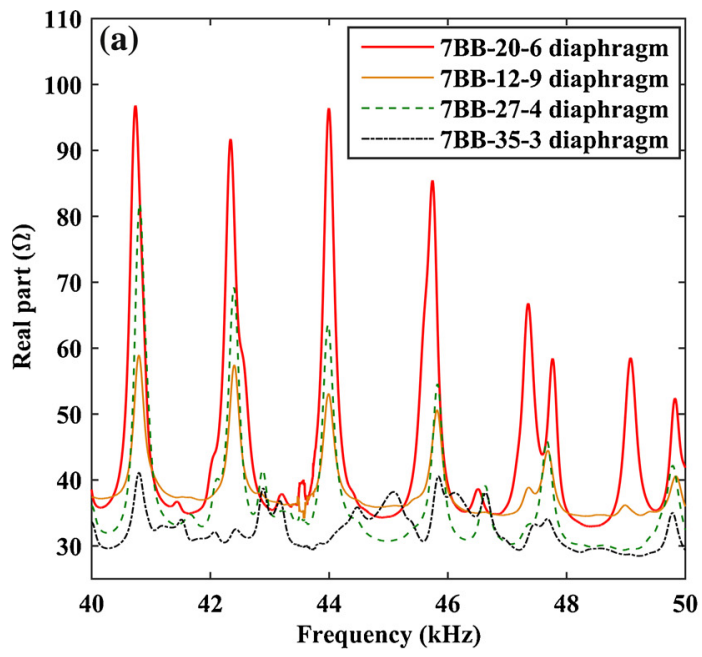

The resonance peaks are more pronounced at low frequencies in the conventional ceramic and less pronounced in the diaphragm. There are also changes in some resonance peaks, probably due to the brass plate. At high frequencies, the two impedance signatures exhibit similar trends, although the resonance peaks are more pronounced in the diaphragm.

The real parts of the impedance signatures of piezoelectric diaphragms of different sizes in the sub-bands of (a) $40-50 \mathrm{kHz}$ and (b) $290-300 \mathrm{kHz}$ are shown in Fig. 8 .

The 7BB-20-6 diaphragm provides an impedance signature with more pronounced peaks than those of the other diaphragms. The 7BB-35-3 diaphragm (the largest diaphragm) produces the least pronounced peaks. At high frequencies, only the 7BB-20-6 diaphragm presents noticeable resonance peaks.

These results reflect the mechanical impedance and static capacitance of the transducer, which depend on transducer size. Small transducers have low mechanical impedance $\left(Z_{P}(\omega)\right)$ in relation to the mechanical impedance of the host structure $\left(Z_{S}(\omega)\right)$ and, according to the results reported in [38] and as per Eq. (3), a high $Z_{S} / Z_{P}$ ratio results in less variation in the electrical impedance $\left(Z_{E}(\omega)\right)$ and, therefore, less pronounced resonance peaks. In contrast, large transducers have a higher static capacitance $\left(C_{0}\right)$ and therefore a lower capacitive reactance $\left(1 / j \omega C_{0}\right)$, which also results

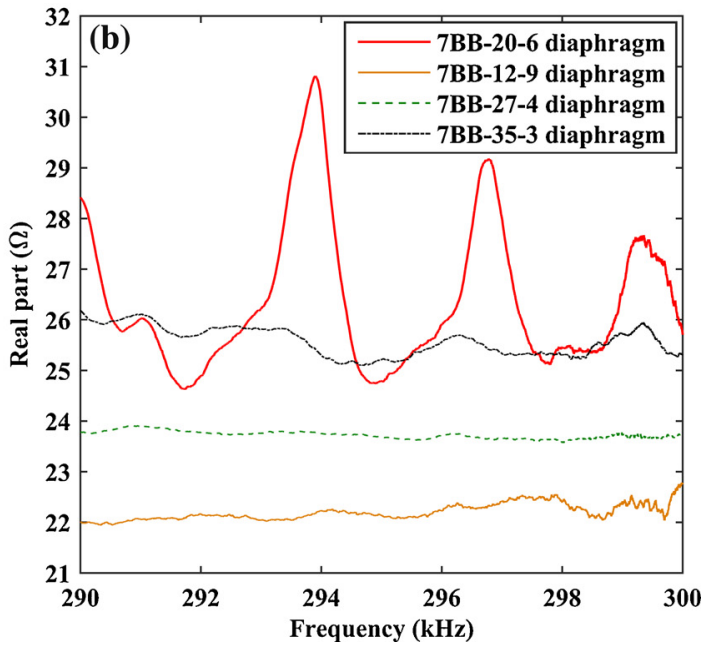

Fig. 8. Real parts of the impedance signatures of diaphragms of different sizes in the two sub-band frequency ranges. 


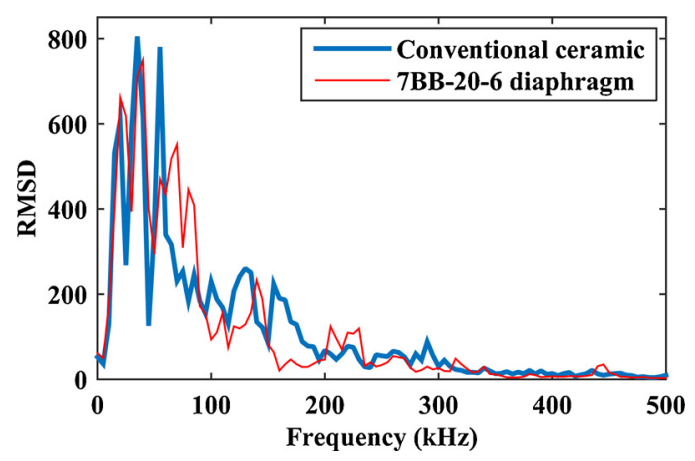

Fig. 9. RMSD indices obtained from the conventional ceramic and the 7BB-20-6 diaphragm.

in smaller resonance peaks, especially at high frequencies $(\omega)$, as can be understood by examining Eq. (1). Therefore, in general, it is expected that small and large transducers provide less significant resonance peaks in their electrical impedance signatures, which is consistent with the results shown in Fig. 8. However, in the particular case of piezoelectric diaphragms, a more detailed analysis requires the development of an appropriate electromechanical model to consider the geometry and the effect of the brass plate, which is beyond the scope of this study.

In addition to pronounced resonance peaks, the transducer must be able to detect changes in these peaks due to the presence of damage. To evaluate the sensitivity of the transducers to damage throughout the frequency range, the RMSD index was calculated using Eq. (4) over the entire frequency range of $0-500 \mathrm{kHz}$ in 5 $\mathrm{kHz}$ sub-bands. Damage was induced in the structures according to the procedure described in Section 4. Fig. 9 shows the RMSD indices obtained from the conventional ceramic and the 7BB-206 diaphragm, and the results obtained for diaphragms of different sizes are shown in Fig. 10.

Both the conventional ceramic and the 7BB-20-6 diaphragm provide RMSD indices with similar trends and amplitudes, presenting alternating bands of higher and lower sensitivity throughout the frequency range. In addition, the indices tend to decrease as the frequency increases. This result is consistent with the PSDs shown in Fig. 5.

As shown in Fig. 10, among the analyzed diaphragms, the 7BB-20-6 diaphragm has the highest RMSD index over the entire frequency range except in some sub-bands and at low frequencies, where the 7BB-27-4 diaphragm showed a higher index. In general, the 7BB-12-9 and 7BB-35-3 diaphragms have lower indices. These results are consistent with the PSDs shown in Fig. 6. As discussed above, it is expected that small and large transducers provide impedance signatures with less pronounced resonance peaks and therefore lower damage indices [38]. However, a detailed theoretical analysis is beyond the scope of this study.

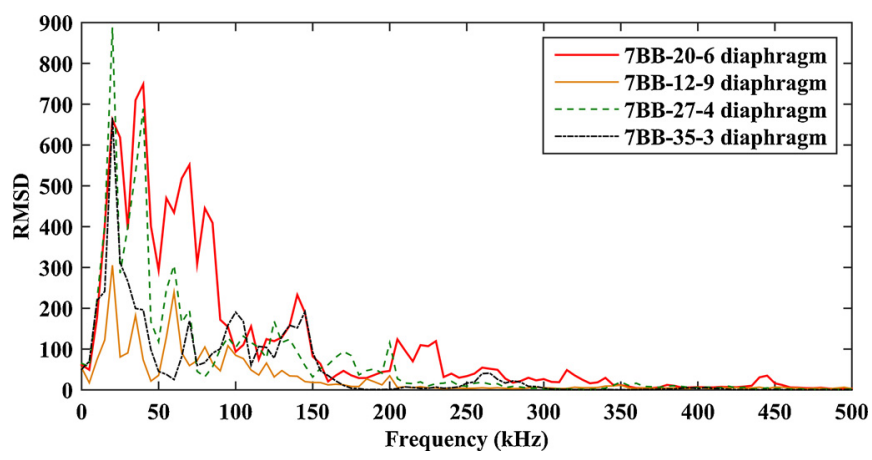

Fig. 10. RMSD indices obtained from the diaphragms of different sizes.
As the EMI method is sensitive to temperature variation [29,37], we analyzed temperature effects, which are presented and discussed in the next section.

\subsection{Temperature effects}

Temperature variations cause shifts in both the frequency and the amplitude of the resonance peaks of the electrical impedance signatures. To analyze and compare the effects of temperature on the signatures obtained from the piezoelectric diaphragms, 26 impedance signatures were measured at temperatures ranging from 20 to $70^{\circ} \mathrm{C}$ at steps of $2{ }^{\circ} \mathrm{C}$ according to the procedure described in Section 4.

The results obtained from the conventional ceramic were compared with those obtained from the 7BB-20-6 diaphragm, which has a piezoelectric element and dimensions similar to those of the ceramic. For example, shifts in the frequency and amplitude can be observed in the resonance peak at approximately $40.5 \mathrm{kHz}$. The real parts of the impedance signatures obtained from the conventional ceramic and the diaphragm are shown in Figs. 11 and 12, respectively.

According to Figs. 11 and 12, the shifts in frequency and amplitude are similar between the two transducers. As the temperature increases, the amplitude and frequency of the resonance peak decrease. The shift in frequency for a temperature increase from 20 to $70^{\circ} \mathrm{C}$ is $-700 \mathrm{~Hz}$ for the conventional ceramic and $-690 \mathrm{~Hz}$ for the diaphragm. With respect to the amplitude of the peaks, the maximum variation is approximately $-23 \Omega(-24 \%)$ for the ceramic and approximately $-10 \Omega(-13 \%)$ for the diaphragm.

For the temperature increase from 20 to $24^{\circ} \mathrm{C}$, the shifts in frequency and amplitude in the signatures obtained from the diaphragm are more uniform than those in the signatures obtained from the conventional ceramic. Uniform shifts with temperature variation are desirable in some compensation methods [29,37].

The results obtained from the other diaphragms are similar to those obtained from the 7BB-20-6 diaphragm; therefore, the impedance signatures of the other diaphragms are not shown. The shifts are also uniform with respect to the temperature variation. The maximum frequency and amplitude shifts were $-620 \mathrm{~Hz}$ and $-35 \%$, respectively, for both the 7BB-12-9 and 7BB-27-4 diaphragms and $-610 \mathrm{~Hz}$ and $-44 \%$, respectively, for the $7 \mathrm{BB}-35-3$ diaphragm.

In addition to the temperature effects, it is important to consider the reproducibility of the impedance signatures obtained from the piezoelectric diaphragms. The results of the long-term reproducibility tests are presented in the next section.

\subsection{Reproducibility of the results}

Because damage detection in the EMI method is usually based on a comparison of two impedance signatures where one is used as a reference, the measuring system and the piezoelectric transducers must show reproducibility in the impedance signatures. Therefore, in the last test, the impedance signatures obtained from the 7BB-20-6 diaphragm were measured every $24 \mathrm{~h}$ for one week and compared with those obtained from the conventional ceramic.

The impedance signatures of the two transducers were obtained at a temperature of $20^{\circ} \mathrm{C}$ using the same measuring system [31] to ensure that any variation in the signatures could be primarily attributed to the transducers. To evaluate the reproducibility of the impedance signatures, the RMSD index was calculated for each signature adopting the first one as the baseline. The indices were calculated in the frequency range of $35-40 \mathrm{kHz}$, which provided a high RMSD index for the two transducers, as shown in Fig. 9. Because the structure is healthy, low RMSD indices are expected, indicating good reproducibility among the signatures. 


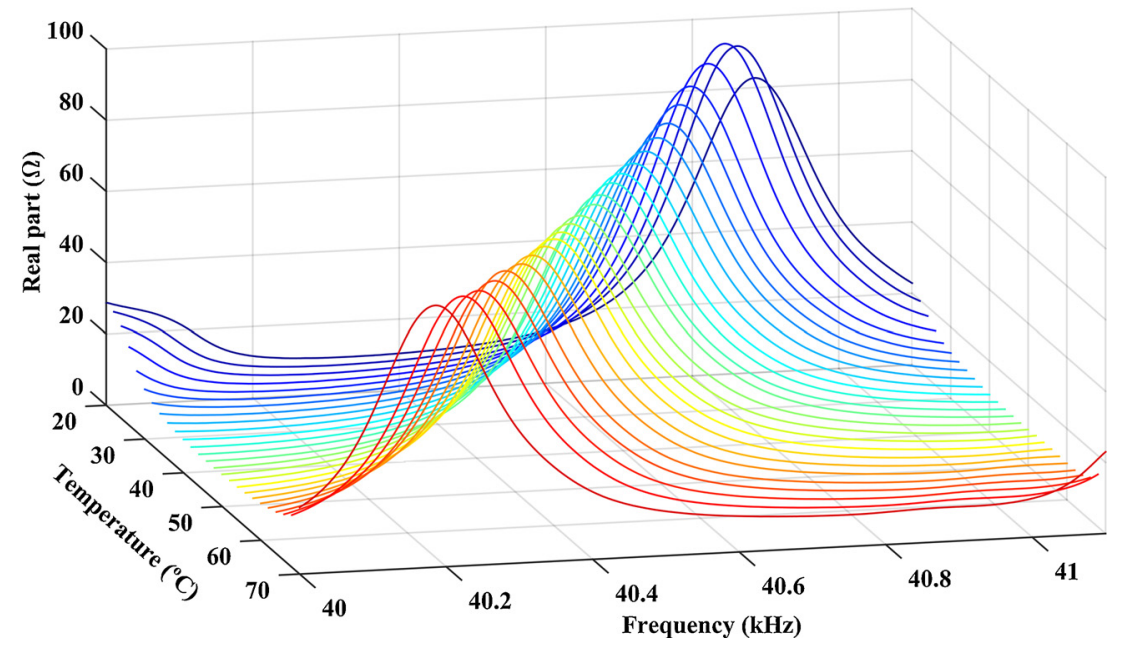

Fig. 11. Temperature effects on the impedance signatures obtained from the conventional ceramic.

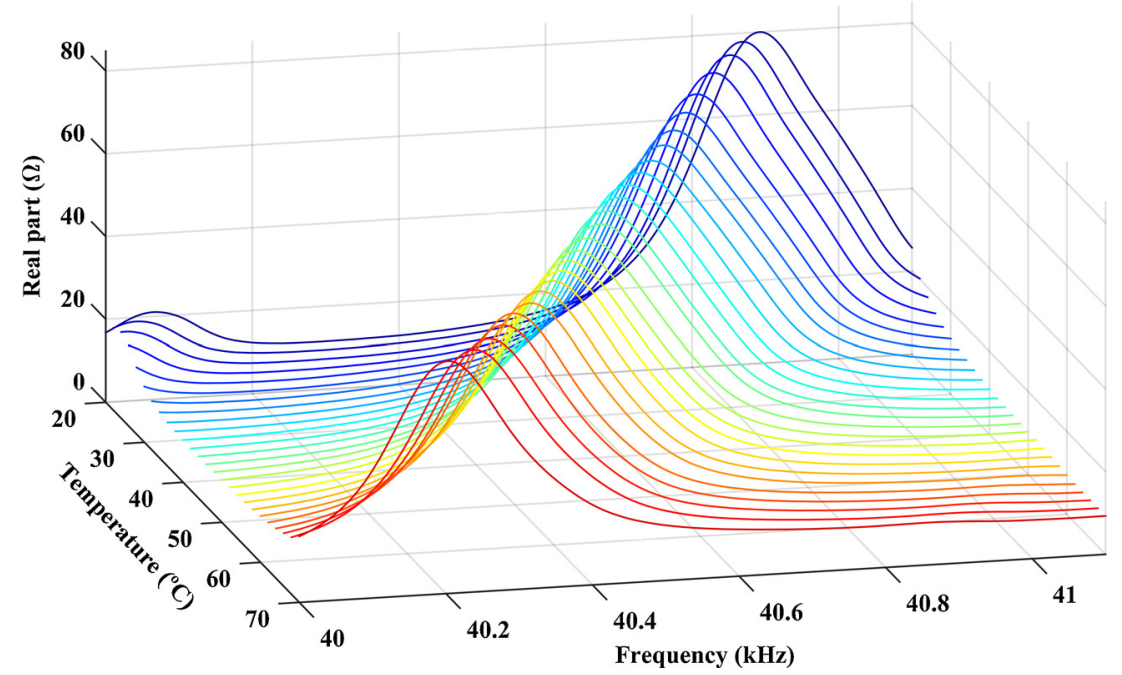

Fig. 12. Temperature effects on the impedance signatures obtained from the 7BB-20-6 diaphragm.
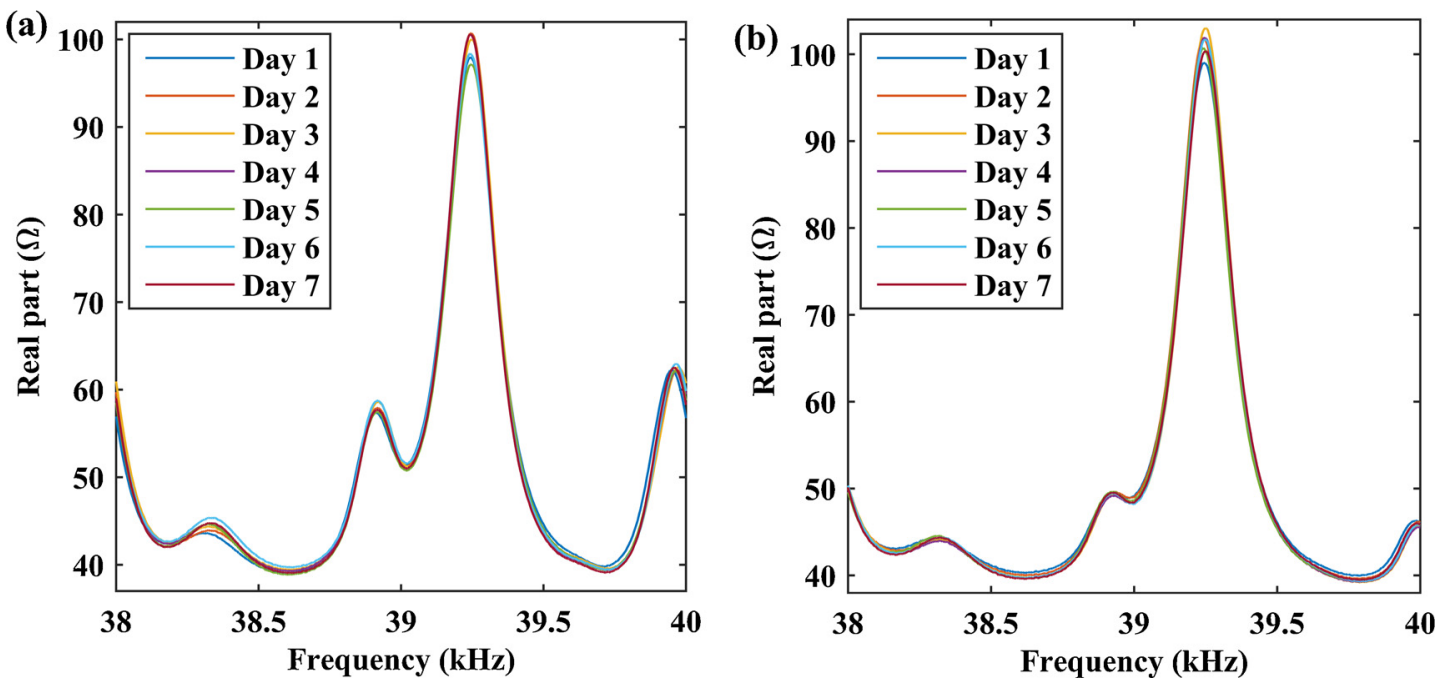

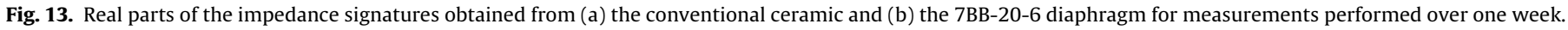




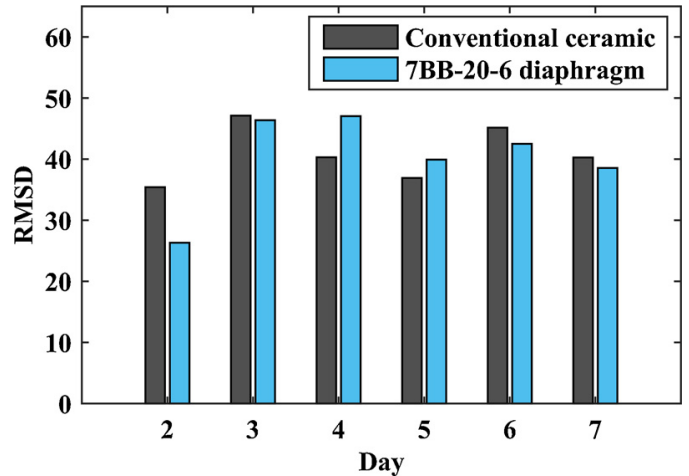

Fig. 14. RMSD indices obtained from the conventional ceramic and the 7BB-20-6 diaphragm for measurements performed over one week.

The real parts of the impedance signatures obtained from the conventional ceramic and the 7BB-20-6 diaphragm for measurements performed over one week are shown in Fig. 13(a) and (b), respectively. A narrow frequency range is displayed to facilitate comparison.

As shown in Fig. 13, the two transducers provide good reproducibility. Some variations were observed in the impedance signatures. However, these variations are small and similar between the two transducers. A comparison of the reproducibility between the two transducers can be accomplished by calculating the RMSD indices. Fig. 14 shows the RMSD indices obtained when using the first impedance signature (Day 1 ) as the baseline.

The indices obtained from the two transducers for the healthy structure are low compared with those obtained for the damaged structure in the same frequency band, as shown in Fig. 9. For the damaged structure, the RMSD indices were greater than 700, whereas for the healthy structure, the indices were lower than 50 , even after the one-week test period, indicating good reproducibility of the results.

\section{Conclusions}

In this study, we presented an experimental analysis of the feasibility of low-cost piezoelectric diaphragms for detecting structural damage based on the electromechanical impedance method.

The experimental results indicated that the piezoelectric diaphragms are able to detect structural damage: They present damage indices with values similar to those of a conventional PZT ceramic of similar size. Furthermore, both transducers showed similar behaviors with respect to temperature effects and the reproducibility of the results.

Therefore, this study supports the use of piezoelectric diaphragms, which are inexpensive and readily available, for research in the field of monitoring systems based on the electromechanical impedance principle. However, these results are only valid under the experimental conditions of the study. The viability of these diaphragms in large or different material structures and involving excitation signals of different types or amplitudes requires further research.

\section{Acknowledgments}

The authors acknowledge the anonymous reviewers for their valuable suggestions and the financial support received from grant \#2015/02500-6, São Paulo Research Foundation (FAPESP).

\section{Appendix A. Supplementary data}

Supplementary data associated with this article can be found, in the online version, at http://dx.doi.org/10.1016/j.sna.2015.11.031.

\section{References}

[1] C.R. Farrar, K. Worden, Structural health monitoring: A machine learning perspective, John Wiley \& Sons Chichester, United Kingdom, 2013 http:// onlinelibrary.wiley.com/book/10.1002/9781118443118.

[2] C. Boller, Structural health monitoring-its association and use, in: W. Ostachowicz, J.A. Güemes (Eds.), New Trends in Structural Health Monitoring, CISM International Centre for Mechanical Sciences, vol. 542, Springer Vienna, Italy, 2013, pp. 1-79 http://dx.doi.org/10.1007/978-3-7091-1390-5_1.

[3] V. Giurgiutiu, Embedded NDT with piezoelectric wafer active sensors, in: O. Güneş, Y. Akkaya (Eds.), Nondestructive Testing of Materials and Structures, RILEM Bookseries, vol. 6, Springer, Netherlands, 2013, pp. 987-992; http://dx.doi.org/10.1007/978-94-007-0723-8_138.

[4] Q. Han, J. Xu, A. Carpinteri, Localization of acoustic emission sources in structural health monitoring of masonry bridge, Struc. Control Health Monit 22 (2015) 314-329 http://dx.doi.org/10.1002/stc.1675.

[5] V. Arjun, B. Sasi, B. Purna Chandra Rao, C.K. Mukhopadhyay, T. Jayakumar, Optimisation of pulsed eddy current probe for detection of sub-surface defects in stainless steel plates, Sens. Actuators A: Phys. 226 (2015) 69-75 http://dx.doi.org/10.1016/j.sna.2015.02.018.

[6] F. Li, H. Peng, G. Meng, Quantitative damage image construction in plate structures using a circular PZT array and lamb waves, Sens. Actuators A: Phys. 214 (2014) 66-73 http://dx.doi.org/10.1016/j.sna.2014.04.016.

[7] J. Zhao, T. Bao, U. Amjad, Optical fiber sensing of small cracks in isotropic homogeneous materials, Sens. Actuators A: Phys. 225 (2015) 133-138 http:// dx.doi.org/10.1016/j.sna.2015.02.017.

[8] D. Roach, Real time crack detection using mountable comparative vacuum monitoring sensors, Smart Struct. Syst. 5 (2009) 317-328 http://smsystems.com.au/_content/documents/686.pdf.

[9] A. Martowicz, M. Rosiek, Electromechanical impedance method, in: T. Stepinski, T. Uhl, W. Staszewski (Eds.), Advance d Structural Damage Detection: From Theory to Engineering Applications, John Wiley \& Sons Chichester, United Kingdom, 2013, pp. 141-176 http://dx.doi.org/10.1002/ 9781118536148.ch6.

[10] V.G.M. Annamdas, C.K. Soh, Application of electromechanical impedance technique for engineering structures: review and future issues, J. Intell. Mater. Syst. Struct. 21 (2010) 41-59 http://dx.doi.org/10.1177/1045389X09352816.

[11] Y.X. Zhang, J.H. Yang, F.H. Xu, J.Z. Chen, EMI-based damage identification for beam structures, Adv. Mater. Res. 1081 (2015) 358-362 http://dx.doi.org/10. 4028/www.scientific.net/AMR.1081.358.

[12] V.G.M. Annamdas, M.A. Radhika, Electromechanical impedance of piezoelectric transducers for monitoring metallic and non-metallic structures: a review of wired, wireless and energy-harvesting methods, J. Intell. Mater. Syst. Struct. 24 (2013) 1021-1042 http://dx.doi.org/10.1177/ $1045389 X 13481254$

[13] Y. Chen, Y. Wen, P. Li, Characterization of PZT ceramic transducer embedded in concrete, Sens. Actuators A: Phys. 128 (2006) 116-124 http://dx.doi.org/10. 1016/j.sna.2006.01.001.

[14] G.M. Annamdas, J.H.L. Pang, Y.X. Chew, H.J. Hoh, K. Zhou, B. Song, Fatigue monitoring of double surface defects using PZT based electromechanical impedance and digital image correlation methods, Adv. Mater. Res. 891-892 (2014) 551-556 http://dx.doi.org/10.4028/www.scientific.net/AMR.891-892. 551.

[15] V. Giurgiutiu, Piezoelectric wafer active sensors for structural health monitoring: state of the art and future directions, in: Proceedings of Conference of Pressure Vessels and Piping Division, Bellevue, Washington, USA, 2010, pp. 1115-1121 http://dx.doi.org/10.1115/PVP2010-25292.

[16] K.-T. Chang, H.-C. Chiang, C.-W. Lee, Design and implementation of a piezoelectric clutch mechanism using piezoelectric buzzers, Sens. Actuators A: Phys. 141 (2008) 515-522 http://dx.doi.org/10.1016/j.sna.2007.10.018.

[17] N. Lamberti, G. Caliano, A.S. Savoia, ACUPAD: a track-pad device based on a piezoelectric bimorph, Sens. Actuators A: Phys. 222 (2015) 130-139 http://dx. doi.org/10.1016/j.sna.2014.10.031.

[18] R. Liang, Q. Wang, High sensitivity piezoelectric sensors using flexible PZT thick-film for shock tube pressure testing, Sens. Actuators A: Phys. 235 (2015) 317-327 http://dx.doi.org/10.1016/j.sna.2015.09.027.

[19] S.-C. Mou, Analytical design of the optimal driving location for a novel ultrasonic actuator by zone-energy method, Res. J. Appl. Sci. Eng. Technol. 4 (2012) 3937-3942 http://maxwellsci.com/jp/abstract. php?jid=RJASET\&no=224\&abs=12.

[20] B. Guiffard, Y. Guichon, H.W. Gundel, Commercial piezoelectric unimorph diaphragm as a magnetic energy harvester, Electron. Lett. 48 (2012) 1196-1198; http://ieeexplore.ieee.org/lpdocs/epic03/wrapper.htm?arnumber=6317234.

[21] C.G. Gonsalez, S. Silva, M.J. Brennan, V. Lopes Jr., Structural damage detection in an aeronautical panel using analysis of variance, Mech. Syst. Signal Proc. 52-53 (2015) 206-216 http://dx.doi.org/10.1016/j.ymssp.2014.04.015. 
[22] E.S. Freitas, F.G. Baptista, An initial study of commercial piezoelectric diaphragms for damage detection based on the electromechanical impedance principle, in: Proceedings of 2st International Electronic Conference on Sensors and Applications, Sciforum, 2015, pp. 1-6 http://dx.doi.org/10.3390/ ecsa-2-E010.

[23] H.A. Tinoco, D.J. Marulanda, Damage identification in active plates with indices based on gaussian confidence ellipses obtained of the electromechanical admittance, J. Nondestr. Eval. 34 (28) (2015) 1-16 http:// dx.doi.org/10.1007/s10921-015-0299-3.

[24] T.H. Ooijevaar, L. Warnet, R. Loendersloot, R. Akkerman, A. Boer, Vibration based damage identification in a composite t-beam utilising low cost integrated actuators and sensors, in: Proceedings of Sixth European Workshop on Structural Health Monitoring, Dresden, Germany, 2012, pp. 232-239 http://purl.utwente.nl/publications/80765.

[25] P.R. Souza, E.G.O. Nobrega, A fault location method using lamb waves and discrete wavelet transform, J. Braz. Soc. Mech. Sci. Eng. 34 (2012) 515-524 http://dx.doi.org/10.1590/S1678-58782012000400013.

[26] A.H. Meitzler et al., IEEE standard on piezoelectricity: an American national standard. New York: IEEE-ANSI, (1988) 66p. (Std, 176.). http://dx.doi.org/10. 1109/IEEESTD.1988.79638.

[27] V.G.M. Annamdas, K.K. Annamdas, Different types of piezoceramic-structure interaction models using electro mechanical impedance technique: a review, in: Proceedings of SPIE 7292, Sensors and Smart Structures Technologies for Civil, Mechanical, and Aerospace Systems, San Diego, California, USA, 2009, pp. 1-12 http://dx.doi.org/10.1117/12.817497.

[28] C. Liang, F.P. Sun, C.A. Rogers, Coupled electro-mechanical analysis of adaptive material systems-determination of the actuator power consumption and system energy transfer, J. Intell. Mater. Syst. Struct. 5 (1994) 12-20 http://dx. doi.org/10.1177/1045389X9400500102.

[29] G. Park, H. Sohn, C.R. Farrar, D.J. Inman, Overview of piezoelectric impedance-based health monitoring and path forward, Shock Vibr. Digest 35 (2003) 451-463 http://hdl.handle.net/10203/18828.

[30] N.E. Cortez, J. Vieira Filho, F.G. Baptista, Design and implementation of wireless sensor networks for impedance-based structural health monitoring using ZigBee and Global System for Mobile Communications, J. Intell. Mater. Syst. Struct. 26 (2015) 1207-1218 http://dx.doi.org/10.1177/ 1045389 X14538532.

[31] F.G. Baptista, J. Vieira Filho, A new impedance measurement system for PZT-based structural health monitoring, IEEE Trans. Instrum. Meas. 58 (2009) 3602-3608 http://dx.doi.org/10.1109/TIM.2009.2018693.

[32] Murata Manufacturing, Piezoelectric Ceramics (PIEZOTITE) Sensors, Catalog No. P19E-9, 2008 http://www.murata.com/.

[33] Piezo Systems Inc. Piezoceramic Properties, Online http://www.piezo.com/ prodsheet3disk5A.html (accessed 31.07.15).

[34] M.G.R. Sause, Investigation of pencil-lead breaks as acoustic emission sources, J. Acoust. Emiss. 29 (2011) 184-196 http://ndt.net/article/jae/papers/29-184. pdf.
[35] V.A.D. Almeida, F.G. Baptista, P.R. Aguiar, Piezoelectric transducers assessed by the pencil lead break for impedance-based structural health monitoring, IEEE Sens. J. 15 (2015) 693-702 http://dx.doi.org/10.1109/JSEN.2014.2352171.

[36] Standard Guide for Determining the Reproducibility of Acoustic Emission Sensor Response, ASTM Standard E976-10, 2010 http://dx.doi.org/10.1520/ E0976-10.

[37] F.G. Baptista, D.E. Budoya, V.A.D. Almeida, J.A.C. Ulson, An experimental study on the effect of temperature on piezoelectric sensors for impedance-based structural health monitoring, Sensors 14 (2014) 1208-1227 http://dx.doi.org 10.3390/s140101208.

[38] F.G. Baptista, J. Vieira Filho, Sizing PZT transducers in impedance-based structural health monitoring, IEEE Sens. J. 11 (2011) 1405-1414.

\section{Biographies}

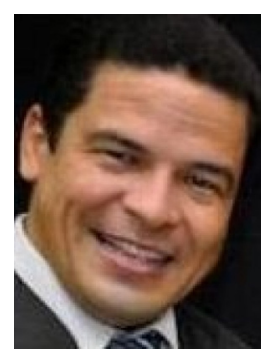

Everaldo Silva de Freitas was born in Presidente Venceslau, Brazil, in 1978. He received a B.S. degree in Computer Engineering from the Centro Universitário de Lins (Unilins), Lins, Brazil, in 2002. Currently, he is a professor of Automation and Electrical Engineering at the Centro Universitário de Lins (Unilins), Lins, Brazil. He is a researcher with the Department of Electrical Engineering, UNESP-Univ Estadual Paulista, Bauru, Brazil, where he is working toward a M.Sc. degree under the advice of Prof Dr. Fabricio Baptista in the field of structural health monitoring based on the electromechanical impedance principle using low-cost piezoelectric transducers.

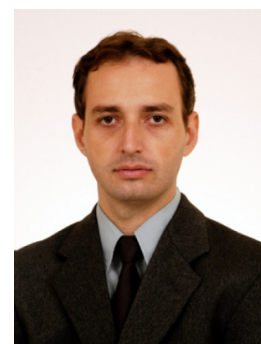

Fabricio Guimarães Baptista received B.S. and Ph.D. degrees in electrical engineering from UNESP-Univ. Estadual Paulista, Ilha Solteira, Brazil, in 2006 and 2010 respectively. From 2010 to 2011, he was a Visiting Scholar at the Center for Intelligent Material Systems and Structures (CIMSS), Virginia Tech, Blacksburg, VA, performing research in the field of structural health monitoring and piezoelectric transducers. He is currently an Assistant Professor in the Department of Electrical Engineering, UNESP, Bauru, where he coordinates the Laboratory of Transducers and Data Acquisition, working in the field of piezoelectricity, structural health monitoring, signal processing, data acquisition, and electronic instrumentation. 